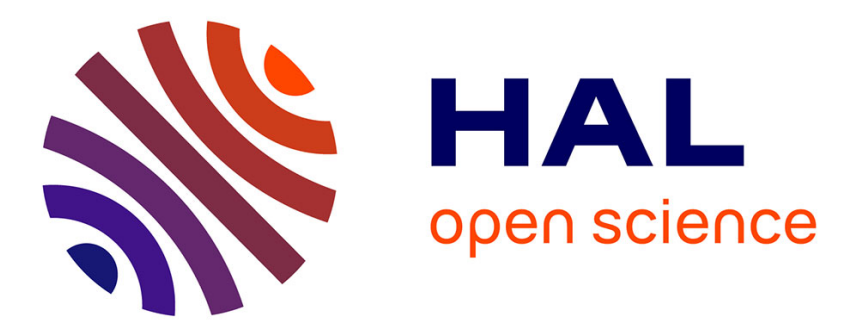

\title{
First identification of the a1Dg2X3Sg electric quadrupole transitions of oxygen in solar and laboratory spectra
}

\author{
I.E. Gordon, Samir Kassi, Alain Campargue, G. C. Toon
}

\section{- To cite this version:}

I.E. Gordon, Samir Kassi, Alain Campargue, G. C. Toon. First identification of the a1Dg2X3Sg electric quadrupole transitions of oxygen in solar and laboratory spectra. Journal of Quantitative Spectroscopy and Radiative Transfer, 2010, 111, pp.1174-1183. 10.1016/j.jqsrt.2010.01.008 . hal00562627

\section{HAL Id: hal-00562627 https://hal.science/hal-00562627}

Submitted on 3 Feb 2011

HAL is a multi-disciplinary open access archive for the deposit and dissemination of scientific research documents, whether they are published or not. The documents may come from teaching and research institutions in France or abroad, or from public or private research centers.
L'archive ouverte pluridisciplinaire HAL, est destinée au dépôt et à la diffusion de documents scientifiques de niveau recherche, publiés ou non, émanant des établissements d'enseignement et de recherche français ou étrangers, des laboratoires publics ou privés. 


\title{
First identification of the $a^{1} \Delta_{\mathrm{g}}-X^{3} \Sigma_{\mathrm{g}}{ }^{-}$electric quadrupole transitions of oxygen in the solar and laboratory spectra
}

\author{
Iouli E. Gordon ${ }^{\mathrm{a}, *}$, Samir Kassi ${ }^{b}$, Alain Campargue ${ }^{b}$, Geoffrey C. Toon $^{\mathrm{c}}$ \\ ${ }^{a}$ Harvard-Smithsonian Center for Astrophysics, Atomic and Molecular Physics Division, \\ Cambridge MA 02138, USA \\ ${ }^{b}$ Université Joseph Fourier/CNRS, Laboratoire de Spectrométrie Physique, \\ 38402 Saint-Martin-d'Hères, FRANCE \\ ${ }^{c}$ Jet Propulsion Laboratory, California Institute of Technology, Pasadena CA 91109, \\ USA
}

*Corresponding author: Tel.: +1 (617) 496-2259;

E-mail address: igordon@ cfa.harvard.edu (I.E. Gordon). 


\begin{abstract}
Electric quadrupole transitions in the $a^{1} \Delta_{\mathrm{g}}-X{ }^{3} \Sigma_{\mathrm{g}}{ }^{-}$band of ${ }^{16} \mathrm{O}_{2}$ near $1.27 \mu \mathrm{m}$ are reported for the first time. They were first detected in atmospheric solar spectra acquired with a ground based Fourier transform spectrometer (FTS) in Park Falls, WI . Subsequently high-sensitivity CW-Cavity Ring Down Spectroscopy (CW-CRDS) experiments were carried out at Grenoble University in the $7717-7917 \mathrm{~cm}^{-1}$ region in order to provide quantitative intensity information for electric quadrupole transitions. Measured intensities were used as input data for calculation of the complete list of electric quadrupole transitions with $\Delta J= \pm 2, \pm 1$ and 0 . The calculation was carried out for the intermediate coupling case and assuming that these transitions are possible only through spin-orbit perturbation of the $\Omega=0$ component of the ground electronic state by $b$ ${ }^{1} \Sigma_{\mathrm{g}}{ }^{+}$state. The calculated line list agrees well with experimental measurements and was used to improve the residuals of the solar atmospheric spectrum. Emission probability for the electric quadrupole band was determined to be $(7.94 \pm 0.80) \times 10^{-7} \mathrm{~s}^{-1}$.
\end{abstract}




\section{Introduction}

Electronic transitions of oxygen have been a subject of over a hundred of theoretical, laboratory and field (atmospheric) studies for over a century. Of particular interest are transitions that involve $X^{3} \Sigma_{\mathrm{g}}{ }^{-}, a^{1} \Delta_{\mathrm{g}}$ and $b^{1} \Sigma_{\mathrm{g}}{ }^{+}$electronic states that arise from the ground electron configuration of oxygen. Since all three of these states have gerade symmetry, only magnetic dipole (M1) and electric quadrupole transitions (E2) are possible between them. The majority of the previous works have reported the magnetic dipole components with an exception of studies of the so-called Noxon band $\left(b^{1} \Sigma_{\mathrm{g}}{ }^{+}-a^{1} \Delta_{\mathrm{g}}\right)$ near $1.91 \mu \mathrm{m}$ [1] that is purely electric quadrupole in nature. Also electric quadrupole transitions within the ground electronic state have been known for a long time [2-4] and are already tabulated in the HITRAN database [5]. Studies of the singlet-triplet electric quadrupole transitions are relatively limited. The $b^{1} \Sigma_{\mathrm{g}}{ }^{+}-X^{3} \sum_{\mathrm{g}}{ }^{-}$electric quadrupole transitions near $760 \mathrm{~nm}$ were first observed by Brault [6] who identified eight lines in the solar spectrum. The first laboratory observation of a single line was reported in the work of Naus et al [7], and very recently Long et al [8] have carried out high-quality quantitative measurements of nine E2 transitions in the A-band using frequency-stabilized cavity ring-down spectroscopy (FS-CRDS) technique. The latter study allowed determination of the band strength of the electric quadrupole component of the A-band which was reported to be equal to only $\sim 8 \times 10^{-6}$ of the magnetic dipole component intensity. No electric quadrupole lines in the $a^{1} \Delta_{\mathrm{g}}-X^{3} \Sigma_{\mathrm{g}}{ }^{-}$band near $1.27 \mu \mathrm{m}$ (from hereon referred as 1Delta band) were identified before. Even sensitive CRDS studies such as those by Newman et al $[9,10]$ did not report the E2 contributions due to their anticipated weakness. Indeed, the theoretical predictions of the emission probability for the E2 component in the classic work of Klotz et al [11] gives value of $5 \times 10^{-7} \mathrm{~s}^{-1}$ which is significantly lower than the value measured for the magnetic dipole component $2.19 \times 10^{-4}$ $\mathrm{s}^{-1}$ [9]. However, even this value (which is underestimated as will be shown in the discussion section) is still larger than the one predicted in the same paper for the quadrupole transitions for the A-band $\left(1.55 \times 10^{-7} \mathrm{~s}^{-1}\right)$. Moreover, Sveshnikova and Minaev [12] have predicted even larger value of $2.3 \times 10^{-6} \mathrm{~s}^{-1}$, although in the later 
publication Minaev and Agren [13], corroborated value from Klotz et al [11]. Thus, considering that 1Delta transitions are located at a lower wavenumber, one would expect their E2 band intensity to be at least an order of magnitude stronger than that of the Aband (see Eq. (19) of Ref. [14]).

In accordance with this logic, we report the identification of electric quadrupole transitions in the 1Delta band in solar atmospheric spectra and their subsequent confirmation in the laboratory using cavity ring down spectroscopy. In addition, an extensive line list of all E2 lines with intensities larger than $10^{-30} \mathrm{~cm} /$ molecule was generated assuming intermediate coupling Hund's case (a-b). This list was used in combination with magnetic dipole line list in order to simulate the solar atmospheric spectrum and yielded improved residuals.

It is our pleasure to contribute this article to the special issue to celebrate Laurence Rothman's jubilee. His contributions to the spectroscopy of oxygen [2, 3, 15-19] are many and invaluable as reference data for atmospheric, planetary and industrial applications. It makes this paper particularly appropriate for the special issue that addition of the oxygen molecule to the AFGL spectral database was Laurence Rothman's first task when he started to work on that project.

\section{First identification in atmospheric solar spectra}

Solar spectra were acquired with a ground based Fourier transform spectrometer (FTS) in Park Falls, WI (460m asl). This instrument is a Bruker IFS125HR belonging to the Total Carbon Column Observing Network (TCCON) [20] (PI; Paul Wennberg, Caltech), as described by Washenfelder et al.[21]. The 7800-8000 $\mathrm{cm}^{-1}$ region containing the $\mathrm{O}_{2}$ absorption band is important for high-accuracy measurements of atmospheric greenhouse gases (e.g., $\mathrm{CO}_{2}, \mathrm{CH}_{4}$ ). This is because ratioing the column abundance of $\mathrm{CO}_{2}$ or $\mathrm{CH}_{4}$ to that of $\mathrm{O}_{2}$, cancels many common systematic errors (e.g. pointing errors, instrument line shape (ILS) uncertainties, zero level offsets). The 1 Delta $\mathrm{O}_{2}$ band has therefore received a great deal of scrutiny from the TCCON community to ensure that the retrieved $\mathrm{O}_{2}$ 
column amounts are free from systematic error. This scrutiny led to the discovery of the missing quadrupole lines which are the subject of this paper.

The spectrum in Figure 1 was measured in December 2004 at a solar zenith angle of $82.45^{\circ}$, thus representing an airmass of 7 . It was cold on the day of the measurement ($20^{\circ} \mathrm{C}$ ) which means that the interfering $\mathrm{H}_{2} \mathrm{O}$ lines are relatively weak, making it easier to see the defects in the $\mathrm{O}_{2}$ spectroscopy. In spectra acquired in summer, the spectral fitting residuals tend to be dominated by $\mathrm{H}_{2} \mathrm{O}$. In Figure 1a the solar spectrum was fitted using latest official release of HITRAN database [5], while in Figure 1b it was fitted using a superior $\mathrm{O}_{2}$ line list provided by Prof. Andrew Orr-Ewing, based on the work of Newman et al [10] with some minor adjustments described in Ref [21]. This list has been used for an update of the HITRAN2008 [5] oxygen line parameters, which is now located on the HITRAN website in the file 07_hit09.par.

\section{Approximate location for Figure 1.}

In Figure $1 \mathrm{~b}$ one can see that there are several lines (most prominently between 7890 and $8000 \mathrm{~cm}^{-1}$ ) that are not accounted for in the reference line lists. These missing lines appear as dips in the residuals. From fits to other spectra measured from Park Falls, we knew that their depth was proportional to the airmass, so we were confident that they originated in the Earth's atmosphere, not the sun's, nor inside the spectrometer. And since they were the same depth year-round, they were not due to a varying gas such as $\mathrm{H}_{2} \mathrm{O}$. Although this pointed towards the missing lines as being $\mathrm{O}_{2}$, it was difficult to explain why their spacing of $16 \mathrm{~cm}^{-1}$ was so large considering that rotational constant of ${ }^{16} \mathrm{O}_{2}$ is $1.4 \mathrm{~cm}^{-1}$. It was therefore certain that these lines were not magnetic dipole oxygen lines.

A previous detection of the electric quadrupole transitions in the solar spectrum of oxygen A band [6], where eight lines were observed with a similar spacing, suggested that the missing 1Delta lines might be electric quadrupole in nature. We therefore used the same program and input constants that were used for calculation of magnetic dipole transitions in HITRAN database [18] to predict the electric quadrupole line positions of the $a^{1} \Delta_{\mathrm{g}}-X^{3} \Sigma_{\mathrm{g}}{ }^{-}$band. The comparison of calculated line positions with the observed 
solar spectrum immediately confirmed that the unidentified lines in Figure $1 \mathrm{~b}$ are in fact electric quadrupole lines. The lines that are apparent from the Figure are $R(1) S(0)$, $T(3) S(4), T(5) S(6), T(7) S(8), T(9) S(10), T(11) S(12)$ and $T(13) S(14)$. Notation of the branches is given as $\Delta N(N) \Delta J(J)$ (see details below). Not all the quadrupole lines give rise to dips in the residuals - those that overlap saturated magnetic dipole absorptions are masked.

It was apparent that in order to fit the solar spectrum with better residuals than those in Figure $1 \mathrm{~b}$ one has to include reliable reference data not only for electric quadrupole line positions but also for their intensities. The intensities that can be estimated directly from the solar spectrum can not be very accurate due to the overlap with interfering absorptions (e.g. $\mathrm{H}_{2} \mathrm{O}, \mathrm{O}_{2}$ magnetic dipole, solar absorptions) and the difficulty of evaluating the effective path length at high solar zenith angles. In order to determine better intensity data, very high-sensitivity CRDS experiments at the Grenoble University were carried out. The measurement of the isolated electric quadrupole lines provided necessary input parameters for calculations of the E2 lines with intensity greater than $10^{-}$ ${ }^{30} \mathrm{~cm}^{-1} /\left(\right.$ molecule $\left.\mathrm{cm}^{-2}\right)$. This calculated line list was then included into the fit of the solar spectrum in Figure 1, yielding improved residuals (Figure 1c). Note that only $\Delta J= \pm 2$ lines were included in the fit to avoid potential correlation of $\Delta J= \pm 1$ and 0 quadrupole lines with corresponding magnetic dipole lines. The details of the laboratory experiments and line list calculations are given below.

\section{Laboratory measurements}

The high-sensitivity absorption spectrum of oxygen was recorded at Grenoble University by CW-Cavity Ring Down Spectroscopy (CW-CRDS) in the 7717-7917 $\mathrm{cm}^{-1}$ region. The fibered distributed feedback (DFB) laser CW-CRDS spectrometer used for these recordings has been described in Refs.[22-24]. Each DFB laser diode has a typical tuning range of about $40 \mathrm{~cm}^{-1}$ by temperature tuning from $-5^{\circ} \mathrm{C}$ to $60^{\circ} \mathrm{C}$. The whole $5850-7917 \mathrm{~cm}^{-1}$ is accessible by using a series of 65 DFB lasers. For the present experiment, six DFB laser diodes were sufficient to cover the $7717-7917 \mathrm{~cm}^{-1}$ region of interest. 
Approximate location for Figure 2.

The electro-polished stainless steel ringdown cell $(l=2 \mathrm{~m}$, inner diameter $\Phi=11$ $\mathrm{mm}$ ) is fitted by a pair of super-mirrors. The reflectivity of the used mirrors (about $99.997 \%$ ) corresponds to empty cell ring down times of about $\tau \sim 200 \mu$ s. About one hundred ringdown events were averaged for each spectral data point; the complete temperature scan of one DFB laser (15000 spectral points) required about 70 minutes. The achieved noise equivalent absorption is about $\alpha_{\min } \sim 4 \times 10^{-11} \mathrm{~cm}^{-1}$ over the whole spectrum. The pressure (measured by an MKS 100 Torr full range capacitance gauge with a $0.1 \%$ accuracy) and the ringdown cell temperature were monitored during the spectrum recording. Fig. 2 shows the overview of the spectrum recorded at a pressure of 50.00 Torr and a temperature of $300.2 \mathrm{~K}$. The spectrum does not cover the high energy part of the $a^{1} \Delta_{\mathrm{g}}-X^{3} \Sigma_{\mathrm{g}}^{-}$band as, to the best of our knowledge, no fibered DFB laser are commercially available above $7917 \mathrm{~cm}^{-1}$.

Each $40 \mathrm{~cm}^{-1}$ wide spectrum recorded with one DFB laser was calibrated independently on the basis of the wavelength values provided by a Michelson-type wavemeter (Burleigh WA-1650, $60 \mathrm{MHz}$ resolution and $100 \mathrm{MHz}$ accuracy). The calibration was further refined by stretching the whole spectrum in order to match accurate positions of reference lines (see Ref. [24] for details). The magnetic dipole line positions of the $a^{1} \Delta_{\mathrm{g}}-X^{3} \Sigma_{\mathrm{g}}{ }^{-}$band of ${ }^{16} \mathrm{O}_{2}$ positions as provided in the recent HITRAN update were used for calibration. The typical uncertainty on the line positions is estimated to be less than $1 \times 10^{-3} \mathrm{~cm}^{-1}$.

The DFB linewidth is about one thousandth of the Doppler broadening leading to a mostly Gaussian line profile. The line centres were determined by using an interactive least squares multi-line fitting program assuming a line profile of Voigt type.

An interactive multi-line fitting program was used to determine the line positions and intensities (http://sourceforge.net/projects/fityk/ version v0.8.6). The complete spectroscopic information retrieved from the recorded spectrum will be presented in a separate contribution. In the present paper, we focus on the quadrupole transitions which were unambiguously identified on the basis of the accurate values of their predicted 
positions. The first step of the line profile fitting consisted in the manual determination of the set of overlapping transitions surrounding the quadrupole transitions that could be fitted independently. At 50.0 Torr, the pressure broadening has a significant contribution to the observed line profile and a Voigt function was adopted for the fitting. The local baseline (assumed to be a linear function of the wavenumber) and the three parameters of each Voigt profile (line centre, integrated absorbance, HWHM of the Lorentzian component) were fitted. The HWHM of the Gaussian component was fixed to its theoretical value $\left(0.00858 \mathrm{~cm}^{-1}\right)$. Overall 16 electric quadrupole transitions with intensities ranging between $7.21 \times 10^{-30}$ and $1.94 \times 10^{-28} \mathrm{~cm} /$ molecule were measured (see Fig. 3). Their measured line positions and intensities are listed in Table 1. Note that as a consequence of frequent blending with much stronger lines it is quite difficult to estimate the experimental uncertainty for intensity measurements and the uncertainties given in Table 1 are crude estimations.

Approximate location for Figure 3.

Approximate location for Table 1.

\section{Theoretical background and line list calculation}

The rotational energy-level diagram of the $a^{1} \Delta_{\mathrm{g}}$ and $X^{3} \Sigma_{\mathrm{g}}{ }^{-}$states of ${ }^{16} \mathrm{O}_{2}$ species is shown in Figure 4 (within Hunds case (b) coupling formalism) together with allowed quadrupole transitions. For ${ }^{16} \mathrm{O}_{2}$ species all rotational levels with total parity (-) are absent in both electronic states which leaves only one $\Lambda$-doubling component of each rotational level in the upper state and only odd rotational levels in the ground state (note that each of the rotational levels in the ground state is split into 3 spin-components, $\mathbf{J}=\mathbf{N}+\mathbf{S}$ ). Quadrupole transitions with $\Delta J= \pm 2, \pm 1$ and 0 are allowed, which leads to 13 possible branches $T(N) S(J), S(N) S(J), R(N) S(J), P(N) O(J), O(N) O(J), N(N) O(J)$ with $\Delta J= \pm 2, S(N) R(J)$, $R(N) R(J), P(N) P(J), O(N) P(J)$ with $\Delta J= \pm 1$ and $R(N) Q(J), Q(N) Q(J), P(N) Q(J)$ with $\Delta J=0$. The $\Delta J= \pm 1$ and 0 transitions coincide with much stronger magnetic dipole transitions and therefore are not observable.

Approximate location for Figure 4. 
The line positions were predicted using the same program and input constants that were used for calculation of magnetic dipole 1Delta transitions in HITRAN database [18].

Significantly more complicated situation is with predicting intensities for the quadrupole transitions. The lower rotational levels of the ground $X^{3} \Sigma_{\mathrm{g}}{ }^{-}$electronic state of oxygen obey intermediate Hund's coupling case. At higher $J$ values spin-uncoupling to the pure case (b) occurs. Original predictions of the infrared quadrupole transitions within the ground state [3] have assumed the pure case (b). However, better agreement with observations was obtained [4] within a frame of intermediate coupling as suggested by Balasubramanian et al [25]. This approach was further extended by Balasubramanian and Narayanan [26] for the E2 transitions in the A band, by combining ideas from Chiu [27] and Watson [28]. We have adapted the framework of Ref. [26] for deriving intensities of the 1Delta E2 transitions.

In the intermediate coupling case the eigenfunctions corresponding to three rotational term series $\mathrm{F}_{i}(J)(i=1,2,3)$ of the $X^{3} \Sigma_{\mathrm{g}}{ }^{-}$state are given by [25]

$$
\begin{aligned}
& \left.\left.\left|F_{1}\right\rangle=\left.s_{J}\right|^{3} \Sigma_{ \pm 1}\right\rangle+\left.c_{J}\right|^{3} \Sigma_{0}\right\rangle \\
& \left.\left|F_{2}\right\rangle=\left.\right|^{3} \Sigma_{ \pm 1}\right\rangle \\
& \left.\left.\left|F_{3}\right\rangle=\left.c_{J}\right|^{3} \Sigma_{ \pm 1}\right\rangle-\left.s_{J}\right|^{3} \Sigma_{0}\right\rangle,
\end{aligned}
$$

where $\mathrm{F}_{1}$ component correspond to $J=N+S$ rotational levels, $\mathrm{F}_{2}$ to $J=N$ and $\mathrm{F}_{3}$ to $J=N-S$. The coefficients $s_{J}$ and $c_{J}$ are given by following equations:

$$
\begin{gathered}
S_{J}=\sqrt{\frac{F_{3}(J)-F_{2}(J)}{F_{3}(J)-F_{1}(J)}}, \\
c_{J}=\sqrt{\frac{F_{2}(J)-F_{1}(J)}{F_{3}(J)-F_{1}(J)}} .
\end{gathered}
$$

In the Hund's case (b) limit $s_{J}=(J+1) /(2 J+1)$ and $c_{J}=J /(2 J+1)$ [28].

The subscripts \pm 1 and 0 in Eq. (1) indicate the values of $\Omega(\Omega=\Lambda+\Sigma)$ of a particular spincomponent in the ground state, where

$\left.\left.\left.\left.\right|^{3} \Sigma_{ \pm 1}\right\rangle=\frac{1}{\sqrt{2}}\left[\left.\right|^{3} \Sigma_{-1}\right\rangle \pm\left.\right|^{3} \Sigma_{1}\right\rangle\right]$ 
Naturally for the upper state the eigenfunction will be given as:

$\left.\left.\left.\left.\right|^{1} \Delta_{ \pm 2}\right\rangle=\frac{1}{\sqrt{2}}\left[\left.\right|^{1} \Delta_{-2}\right\rangle \pm\left.\right|^{1} \Delta_{2}\right\rangle\right]$

The line strength formula for electric quadrupole transitions involving states with nonzero electron spin was derived by Balasubramanian et al [25] as

$S\left(J^{\prime}, J^{\prime \prime}\right)=\left[\frac{2\left(2 J^{\prime \prime}+1\right)}{3}\right] \mid \sum_{\Omega^{\prime \prime} \Omega^{\prime} \lambda} a_{\Omega^{\prime \prime}} a_{\Omega^{\prime}}^{*}\left\langle n^{\prime} \Lambda^{\prime} S^{\prime} \Sigma^{\prime}, v^{\prime}\left|\tilde{Q}_{2 \lambda}\right| n^{\prime \prime} \Lambda^{\prime \prime} S^{\prime \prime} \Sigma^{\prime \prime}, v^{\prime \prime}\right\rangle$

$\times\left. C\left(J^{\prime \prime} 2 J^{\prime}, \Omega^{\prime \prime} \lambda \Omega^{\prime}\right)\right|^{2}$,

where $a_{\Omega}$ are linear coefficients for the linear combination of the intermediate coupling case basis functions. The purely orbital operator $Q_{2 \lambda}(\lambda= \pm 2, \pm 1$ and 0$)$ is a moleculefixed spherical component of the E2 operator and $C\left(J^{\prime \prime} 2 J^{\prime}, \Omega^{\prime \prime} \lambda \Omega^{\prime}\right)$ is a Clebsch-Gordon coefficient. The normal selection rules for the electric quadrupole transitions are $\Delta \Lambda= \pm 2$, \pm 1 and 0 and $\Delta S=0$ [11], therefore the observed E2 transitions are possible only due to perturbations, since $S^{\prime}=0$ and $S^{\prime \prime}=1$. In this case there is a spin-orbit perturbation of the $\Omega=0$ component of the ground state by $b^{1} \Sigma_{\mathrm{g}}{ }^{+}$, and of the $\Omega= \pm 1$ components by a much higher lying ${ }^{1} \Pi$ state. The later contribution is usually neglected [11-13], and we have decided to do the same in this work for simplicity. In Figure 4 transitions that involve $\left|\mathrm{F}_{2}\right\rangle$ component of the ground state are shown as dashed lines, because they are purely $\Omega= \pm 1$ and are not considered in the calculation of the line list. Nevertheless, it is worth noting that this will introduce a small error. The reason for that is that we did tentatively observed $O(N) O(J)$ and $S(N) S(J)$ transitions which are weaker by about two orders of magnitude than other $\Delta J= \pm 2$ transitions. We are working on the more extensive experimental effort that will include accurate measurements of $O(N) O(J)$ and $S(N) S(J)$ transitions and more lines from other branches than the ones reported here, that work will also include the contribution of $\Omega= \pm 1$ in the theoretical treatment [29]. Therefore, only $Q_{20}$ matrix element is included into Eq. (5) in the frame of the current work. Using appropriate Clebsch-Gordon coefficients [30] and Eqs. (1) and (5) following expressions for the line strengths $S\left(J^{\prime}, J^{\prime}\right)$ were derived for each branch represented by solid arrows in Figure 3. 


$$
\begin{array}{ll}
T(N) S(J): & Q_{20}^{2} c_{J}[(J+3)(J+4) / 3(2 J+3)] \\
R(N) S(J): & Q_{20}^{2} s_{J}[(J+3)(J+4) / 3(2 J+3)] \\
P(N) O(J): & Q_{20}^{2} c_{J}[(J-2)(J-3) / 3(2 J-1)] \\
N(N) O(J): & Q_{20}^{2} s_{J}[(J-2)(J-3) / 3(2 J-1)] \\
S(N) R(J): & Q_{20}^{2} c_{J}[2(J+3) / 3] \\
O(N) P(J): & Q_{20}^{2} s_{J}[2(J-2) / 3] \\
R(N) Q(J): & Q_{20}^{2} c_{J}[2(2 J+1)(J+2)(J-1) /(2 J-1)(2 J+3)] \\
P(N) Q(J): & Q_{20}^{2} s_{J}[2(2 J+1)(J+2)(J-1) /(2 J-1)(2 J+3)]
\end{array}
$$

It is not surprising that the E2 line strengths formulae derived here are identical to the ones derived for the ${ }^{1} \Delta-{ }^{1} \Sigma$ transitions in Table 1 of Ref. [27] multiplied by corresponding $c_{J}$ or $s_{J}$ coefficients.

The line intensities in HITRAN units $\left(\mathrm{cm}^{-1} /\left(\right.\right.$ molecule $\left.\left.\mathrm{cm}^{-2}\right)\right)$ were calculated using the following equation [3]:

$S=C Q_{20}^{2} v^{3} S\left(J^{\prime} ; J^{\prime \prime}\right) \exp \left(-c_{2} E^{\prime \prime} / T\right)\left[1-\exp \left(-c_{2} v / T\right)\right]$

where $c_{2}$ is the second radiation constant, $\mathrm{T}=296 \mathrm{~K}$ is the standard temperature used in HITRAN, $E^{\prime \prime}$ is the lower state energy, and $v$ is the wavenumber of the transition.

The value of $C Q^{2}$ was chosen to satisfy the measured intensities of 2 experimental lines $R(1) S(0)$ and $N(13) O(12)$ that had the smallest experimental uncertainty. The experimental intensity was converted from measured value at $300 \mathrm{~K}$ to the one at $296 \mathrm{~K}$. It is worth noting that only for $R(1) S(0)$ line the difference between intensities at these two temperatures exceeds $1 \%$. The $R(1) S(0)$ line was given a highest weight because it is a well isolated strong line, see Figure 2. In addition it is the only electric quadrupole line with $\Delta J= \pm 2$ that involve only $\Omega=0$ component of the ground state, is insensitive to which Hund's coupling case is assumed and therefore is a perfect input parameter for the chosen model.

In Figure 3 the predicted electric quadrupole lines with $\Delta J= \pm 2$ are shown, together with experimental results (recall that we neglected $S(N) S(J)$ and $O(N) O(J)$ lines and therefore they are not shown). The agreement is quite satisfactory; all the predicted intensities are within experimental uncertainties from measured values. Predicted lines with $\Delta J= \pm 2$ that are stronger than $10^{-30} \mathrm{~cm}^{-1} /\left(\right.$ molecule $\left.\mathrm{cm}^{-2}\right)$ are also given in Table 2. The complete line 
list for quadrupole transitions that include transitions with $\Delta J= \pm 2, \pm 1$ and 0 is given in the supplementary file. Considering that we have neglected the contribution from the $\Omega= \pm 1$ components in the ground state and experimental uncertainties of the input values the intensities of the lines are expected to have uncertainty of $5-10 \%$, while the line positions should be reliable to $0.001 \mathrm{~cm}^{-1}$.

Approximate location for Table 2.

\section{Discussion}

The electric quadrupole $a^{1} \Delta_{\mathrm{g}}-X^{3} \Sigma_{\mathrm{g}}{ }^{-}$transitions observed in this work have shown that their intensities, while still being quite weak in comparison with magnetic dipole transitions, are not negligible as was previously thought. Indeed the predicted (previous section) integrated intensity of all $\Delta J= \pm 2, \pm 1$ and 0 transitions of the E2 band given in supplementary material is $(1.13 \pm 0.11) \times 10^{-26} \mathrm{~cm}^{-1} /\left(\right.$ molecule $\left.\mathrm{cm}^{-2}\right)$ while integrated intensity of the magnetic dipole band is $(3.10 \pm 0.10) \times 10^{-24} \mathrm{~cm}^{-1} /\left(\right.$ molecule $\left.\mathrm{cm}^{-2}\right)$ [9]. Therefore the ratio of magnetic dipole to electric quadrupole contributions to the $a^{1} \Delta_{\mathrm{g}}$ $X^{3} \Sigma_{\mathrm{g}}{ }^{-}$band is only $\sim 275$ while for the $A$ band it is $\sim 120,000$ [8]. This implies that despite that magnetic dipole intensities of the $A$ band being much stronger than those of 1Delta band, the electric quadrupole intensities of the $A$ band are in fact weaker. From the integrated band intensity one can derive total emission probability (Einstein A coefficient) [31] which in this case is equal to $(7.94 \pm 0.80) \times 10^{-7} \mathrm{~s}^{-1}$. The theoretical calculations of Klotz et al [11] gives value of $5 \times 10^{-7} \mathrm{~s}^{-1}$. One can note that Equation $(7 \mathrm{c})$ of Ref. [11] (which was used there to calculate emission probability) is missing a factor of (3/2), which is needed to reflect relative degeneracy in the $X^{3} \Sigma_{\mathrm{g}}{ }^{-}$and $a^{1} \Delta_{\mathrm{g}}$ states [9, 31]. If the factor of (3/2) is introduced, the theoretical Einstein A coefficient becomes equal to $7.5 \times 10^{-7} \mathrm{~s}^{-1}$, a value that is remarkably close to the one obtained in this work. Total emission probability of the E2 band in the A band can be derived from the integrated band intensity $(1.8 \pm 0.1) \times 10^{-27} \mathrm{~cm}^{-1} /\left(\right.$ molecule $\left.\mathrm{cm}^{-2}\right)$ reported by Long et al [8] and is equal to $(7.0 \pm 0.1) \times 10^{-7} \mathrm{~s}^{-1}$. The corresponding value from Klotz et al [11] is 
$1.55 \times 10^{-7} \mathrm{~s}^{-1}$ which also misses a factor accounting for relative degeneracy of the $X^{3} \Sigma_{\mathrm{g}}{ }^{-}$ and $b^{1} \Sigma_{\mathrm{g}}{ }^{+}$states, which in this case is a factor of 3 . Although the resulting theoretical value of $4.65 \times 10^{-7} \mathrm{~s}^{-1}$ is smaller than the value derived from integrated intensity from Ref. [8] it still shows fairly good agreement. Finally it is worth noting that the emission probability of the electric quadrupole $a^{1} \Delta_{\mathrm{g}}-X^{3} \Sigma_{\mathrm{g}}{ }^{-}$transitions is much smaller than the one of the spin-allowed by electric quadrupole selection rules. $b^{1} \Sigma_{\mathrm{g}}{ }^{+}-a^{1} \Delta_{\mathrm{g}}$ transitions, which is most recently measured to be $(1.20 \pm 0.25) \times 10^{-3} \mathrm{~s}^{-1}$ [32] .

The predicted line list shows that electric quadrupole transitions with $\Delta J= \pm 1$ and 0 are slightly stronger than those with $\Delta J= \pm 2$. Indeed as it was already mentioned the integrated intensity of all allowed E2 transitions is $(1.13 \pm 0.11) \times 10^{-26} \mathrm{~cm}^{-1} /$ (molecule $\mathrm{cm}^{-}$ ${ }^{2}$ ), however, the integrated intensity of only $\Delta J= \pm 2$ lines is just $(2.18 \pm 0.22) \times 10^{-27} \mathrm{~cm}^{-}$ $1 /\left(\right.$ molecule $\left.\mathrm{cm}^{-2}\right)$. The $\Delta J= \pm 1$ and 0 transitions cannot be observed, due to their coincidence with corresponding magnetic dipole lines. Nevertheless, they can introduce up to $1.5 \%$ error into intensities of magnetic dipole lines. It will be interesting in the future to reevaluate results from previous magnetic dipole transition measurements (such as the ones in Ref. [9]) for consistency between the branches that coincide with those of electric quadrupole transitions and those that do not.

\section{Conclusion}

The electric quadrupole $a^{1} \Delta_{\mathrm{g}}-X^{3} \Sigma_{\mathrm{g}}{ }^{-}$transitions were observed and identified for the first time in ground-based solar absorption spectra, thanks to a long absorption path through the atmosphere and a good line list representing the $\mathrm{O}_{2}$ magnetic dipole transitions. In order to provide an accurate reference data to fit the quadrupole transitions observed in the solar spectrum, high-sensitivity CRDS experiments were carried out at the Grenoble University under controlled conditions. Experiments have confirmed the assumption that the contribution from the $\Omega= \pm 1$ component of the ground electronic state is insignificant in comparison with that from $\Omega=0$ component. Intensities of two of the stronger quadrupole transitions, well isolated from the magnetic dipole 
lines, were measured and used to derive the input parameters for calculation of the complete electric quadrupole line list. The line list was then used to fit the solar spectrum yielding an improvement. The Einstein A coefficient derived in this work agrees well with the theoretical prediction by Klotz et al [11] corrected by a factor of 3/2. Strongest $\Delta J= \pm 2$ quadrupole transitions will be added to the next edition of the HITRAN database.

\section{Acknowledgments}

The authors are grateful to Rebecca Washenfelder and Jean-François Blavier who set up the TCCON FTS at Park Falls, WI, and recorded the atmospheric solar absorption spectra used here. We acknowledge Paul Wennberg for the use of these data and the NASA Carbon Cycle program which funds TCCON. We also thank Andrew Orr-Ewing for providing such a good $\mathrm{O}_{2}$ magnetic dipole linelist, that the missing quadrupole lines became so prominent. We also thank Robert Field and Charles Miller for fruitful discussions and Laurence Rothman and Robert Gamache for providing input parameters and program for HITRAN line positions calculation. The HITRAN database managed for over 35 years by Dr. Rothman is an invaluable help in many fields and all of the authors have benefited from using it many times and were honored to contribute to it or validate its data. Part of this work was performed at the Jet Propulsion Laboratory, , California Institute of Technology, under contract with NASA and at Grenoble University under the ANR project "IDEO". 


\section{References}

[1] Noxon JF. Observation of the $(0,0)$ Band of the ${ }^{1} \Sigma_{\mathrm{g}}{ }^{-}-{ }^{1} \Delta_{\mathrm{g}}$ System of Oxygen in the Day and Twilight Airglow. Nature 1962;196:157-8.

[2] Goldman A, Reid J, Rothman LS. Identification of electric quadrupole $\mathrm{O}_{2}$ and $\mathrm{N}_{2}$ lines in the infrared atmospheric absorption spectrum due to the vibration-rotation fundamentals. Geophys Res Lett 1981;8:77.

[3] Rothman LS, Goldman A. Infrared electric quadrupole transitions of atmospheric oxygen. Appl Opt 1981;20:2182-4.

[4] Goldman A, Rinsland CP, Canova B, Zander R, Dangnhu M. Improved spectral parameters for the ${ }^{16} \mathrm{O}_{2}$ infrared forbidden lines in the $X^{3} \Sigma_{\mathrm{g}}{ }^{-}(0-1)$ band. JQSRT 1995;54:757-65.

[5] Rothman LS, Gordon IE, Barbe A, Benner DC, Bernath PF, Birk M, et al. The HITRAN 2008 molecular spectroscopic database. Journal of Quantitative Spectroscopy and Radiative Transfer 2009;110:533-72.

[6] Brault JW. Detection of electric quadrupole transitions in the oxygen A band at 7600 Å. J Mol Spectrosc 1980;80:384-7.

[7] Naus H, de Lange A, Ubachs W. $b^{1} \Sigma_{\mathrm{g}}{ }^{+}-\mathrm{X}^{3} \Sigma_{\mathrm{g}}{ }^{-}(0,0)$ band of oxygen isotopomers in relation to tests of the symmetrization postulate in ${ }^{16} \mathrm{O}_{2}$. Physical Review A 1997;56:4755-63.

[8] Long DA, Havey DK, Okumura M, Pickett HM, Miller CE, Hodges JT. Laboratory measurements and theoretical calculations of $\mathrm{O}_{2} \mathrm{~A}$ band electric quadrupole transitions. Phys Rev A 2009;80:042513-12.

[9] Newman SM, Lane IC, Orr-Ewing AJ, Newnham DA, Ballard J. Integrated absorption intensity and Einstein coefficients for the $\mathrm{O}_{2} a^{1} \Delta_{\mathrm{g}}-X^{3} \Sigma_{\mathrm{g}}{ }^{-}(0,0)$ transition: A comparison of cavity ringdown and high resolution Fourier transform spectroscopy with a long-path absorption cell. J Chem Phys 1999;110:10749-57.

[10] Newman SM, Orr-Ewing AJ, Newnham DA, Ballard J. Temperature and pressure dependence of line widths and integrated absorption intensities for the $\mathrm{O}_{2} a^{1} \Delta_{\mathrm{g}}-X^{3} \Sigma_{\mathrm{g}}$ $(0,0)$ transition. J Phys Chem A 2000;104:9467-80.

[11] Klotz R, Marian CM, Peyerimhoff SD, Hess BA, Buenker RJ. Calculation of spin-forbidden radiative transitions using correlated wavefunctions: Lifetimes of $b^{1} \Sigma^{+}$, $a^{1} \Delta$ states in $\mathrm{O}_{2}, \mathrm{~S}_{2}$ and SO. Chem Phys 1984;89:223-36.

[12] Sveshnikova EB, Minaev BF. Mechanism of the nonradiative ${ }^{1} \Delta_{\mathrm{g}}-{ }^{3} \Sigma_{\mathrm{g}}{ }^{-}$transition in molecular oxygen in solution. Optika I Spektroskopiya 1983;54:542-5.

[13] Minaev BF, Agren H. Collision-induced $b^{1} \Sigma_{\mathrm{g}}{ }^{+}-a^{1} \Delta_{\mathrm{g}}, b^{1} \Sigma_{\mathrm{g}}{ }^{+}-X^{3} \Sigma_{\mathrm{g}}{ }^{-}$and $a^{1} \Delta_{\mathrm{g}}-X$ ${ }^{3} \Sigma_{\mathrm{g}}{ }^{-}$transition probabilities in molecular oxygen. Journal of the Chemical Society-

Faraday Transactions 1997;93:2231-9.

[14] Simecková M, Jacquemart D, Rothman LS, Gamache RR, Goldman A. Einstein $A$-coefficients and statistical weights for molecular absorption transitions in the HITRAN database. JQSRT 2006;98:130-55.

[15] Rothman LS. Update of the AFGL atmospheric absorption line parameters compilation. Appl Opt 1978;17:3517. 
[16] Rothman LS. Magnetic dipole infrared atmospheric oxygen bands. Appl Opt 1982;21:2428-31.

[17] Rothman LS, Gamache RR, Barbe A, Goldman A, Gillis JR, Brown LR, et al. AFGL atmospheric absorption line parameters compilation - 1982 edition. Appl Opt 1983;22:2247-56.

[18] Gamache RR, Goldman A, Rothman LS. Improved spectral parameters for the three most abundant isotopomers of the oxygen molecule. 1998:495-509.

[19] Goldman A, Stephen TM, Rothman LS, Giver LP, Mandin JY, Gamache RR, et al. The $1-\mu \mathrm{m} \mathrm{CO} 2$ bands and the $\mathrm{O}_{2}(0-1) X^{3} \Sigma_{\mathrm{g}}{ }^{-}-a^{1} \Delta_{\mathrm{g}}$ and (1-0) $X^{3} \Sigma_{\mathrm{g}}{ }^{-}-b^{1} \Sigma_{\mathrm{g}}{ }^{+}$bands in the Earth atmosphere. JQSRT 2003;82:197-205.

[20] http://www.tccon.caltech.edu/index.html

[21] Washenfelder RA, Toon GC, Blavier J-F, Yang Z, Allen NT, Wennberg PO, et al. Carbon dioxide column abundances at the Wisconsin Tall Tower site. Journal of Geophysical Research (Atmospheres) 2006;111:22305.

[22] Macko P, Romanini D, Mikhailenko SN, Naumenko OV, Kassi S, Jenouvrier A, et al. High sensitivity $\mathrm{CW}$-cavity ring down spectroscopy of water in the region of the 1.5mum atmospheric window. J Mol Spectrosc 2004;227:90-108.

[23] Morville J, Romanini D, A.A. K, M. C. Two schemes for trace detection using cavity ringdown spectroscopy. Appl Phys 2004;D78:465-76.

[24] Perevalov BV, Kassi S, Romanini D, Perevalov VI, Tashkun SA, Campargue A. $\mathrm{CW}$-cavity ringdown spectroscopy of carbon dioxide isotopologues near $1.5 \mu \mathrm{m}$. J Mol Spectrosc 2006;238:241-55.

[25] Balasubramanian TK, D'Cunha R, Rao KN. Line strengths in a ${ }^{3} \Sigma-{ }^{3} \Sigma$ quadrupole transition with intermediate coupling: Application to line intensities in the quadrupole fundamental band of the oxygen molecule. J Mol Spectrosc 1990;144:374-80. [26] Balasubramanian T, Narayanan O. Magnetic dipole and electric quadrupole line strengths for the atomospheric oxygen $\left(b^{1} \Sigma_{\mathrm{g}}{ }^{+}-\mathrm{X}^{3} \Sigma_{\mathrm{g}}{ }^{-}\right)$system. Acta Physica Hungarica 1994;74:341-53.

[27] Chiu Y-N. Electric-Quadrupole and Magnetic-Dipole Radiation in Linear Molecules. Applications to ${ }^{1} \Pi-{ }^{3} \Pi$ Transitions. The Journal of Chemical Physics 1965;42:2671-81.

[28] Watson JKG. Rotational line intensities in ${ }^{3} \Sigma-{ }^{1} \Sigma$ electronic transitions. Can J Phys 1968;46:1637-43.

[29] Kassi S, Wang L, Campargue A, Field RW, Rothman LS, Gordon IE. Weak magnetic dipole and electric quadrupole transitions of oxygen at $1.27 \mu \mathrm{m}$, unpublished work.

[30] Condon EU, Shortley GH, The Theory of Atomic Spectra. 6 ed.; Cambridge University Press: 1963.

[31] Gamache RR, Goldman A. Einstein A coefficient, integrated band intensity, and population factors: application to the $a^{1} \Delta_{\mathrm{g}}-X^{3} \Sigma_{\mathrm{g}}{ }^{-}(0,0) \mathrm{O}_{2}$ band. JQSRT 2001;69:389401.

[32] Vagin NP, Ionin AA, Kochetov IV, Napartovich AP, Podmar'kov YP, Frolov MP, et al. Measurement of the $\mathrm{O}_{2} b^{1} \Sigma_{\mathrm{g}}{ }^{+}-a^{1} \Delta_{\mathrm{g}}$ transition probability by the method of intracavity laser spectroscopy. Quantum Electronics 2005;35:378-84. 


\section{Figure captions.}

Figure 1. Solar FTS spectrum of the $a^{1} \Delta_{\mathrm{g}}-X^{3} \Sigma_{\mathrm{g}}{ }^{-}$band of oxygen measured in December 2004 from Park Falls, Wisconsin (442 m asl) at a solar zenith angle of $82.45^{\circ}$. a) Fitted usingthe HITRAN2008 file of magnetic dipole transitions; b) Fitted using updated HITRAN2008 file (07_hit09.par) of magnetic dipole transitions based on the work of Orr-Ewing; c) adding the calculated electric quadrupole line list. In each panel, the black diamond symbols represent the measured spectrum, and the black line represents the calculation. The residual trace at the top of each panel represents their difference (Measured-Calculated). The various colored lines represent the various contributions to the calculated transmittance. The $\mathrm{O}_{2}$ collision induced absorption (orange curve) is fitted separately from the discrete $\mathrm{O}_{2}$ absorption (red curve). Note that the RMS residual in panel c) is almost a factor 2 better than that in panel a). The spectrum, acquired in $75 \mathrm{~s}$ of integration time, was one of over 300 measured on this day. It represents an effective atmospheric path length of over $50 \mathrm{~km}$.

Figure 2. Overview of the $a^{1} \Delta_{\mathrm{g}}-X^{3} \Sigma_{\mathrm{g}}{ }^{-}$band of oxygen recorded by CW-CRDS positions $(P=50.0$ Torr, $T=300.2 \mathrm{~K})$. The two enlargements show the $N(13) O(12)$ and $R(1) S(0)$ electric dipole transitions lying between the much stronger magnetic dipole transitions.

Figure 3. Predicted and observed intensities of the electric quadrupole transitions with $\Delta J= \pm 2$. Transitions that involve $J^{\prime \prime}=N^{\prime \prime}$ levels are not shown, as they were ignored within the model applied in calculation. Quantum identifications of few lines are shown as reference points.

Figure 4. The energy level diagram of the $X^{3} \Sigma_{\mathrm{g}}{ }^{-}$and $a^{1} \Delta_{\mathrm{g}}$ states of ${ }^{16} \mathrm{O}_{2}$. Allowed electric quadrupole transitions are shown with arrows. Dashed arrows indicate transitions from the spin-component of the ground state that is purely $|\Omega|=1$ in nature. The $\Lambda$ doubling of the rotational levels of the excited state is not shown, because for each rotational level one of the components is missing in ${ }^{16} \mathrm{O}_{2}$. 
Table 1. Experimental line positions and intensities.

\begin{tabular}{ccc}
\hline$v, \mathrm{~cm}^{-1}$ & $\begin{array}{c}\mathrm{S}, \\
\mathrm{cm} / \mathrm{molecule}\end{array}$ & Assignment \\
\hline 7723.922 & $1.15(10) \mathrm{E}-29$ & $\mathrm{~N}(19) \mathrm{O}(18)$ \\
7760.599 & $2.69(49) \mathrm{E}-29$ & $\mathrm{~N}(15) \mathrm{O}(14)$ \\
7778.728 & $2.93(30) \mathrm{E}-29$ & $\mathrm{~N}(13) \mathrm{O}(12)$ \\
7796.716 & $2.80(34) \mathrm{E}-29$ & $\mathrm{~N}(11) \mathrm{O}(10)$ \\
7814.558 & $2.90(35) \mathrm{E}-29$ & $\mathrm{~N}(9) \mathrm{O}(8)$ \\
7816.000 & $7.21(14) \mathrm{E}-30$ & $\mathrm{P}(23) \mathrm{O}(24)$ \\
7832.258 & $1.89(22) \mathrm{E}-29$ & $\mathrm{~N}(7) \mathrm{O}(6)$ \\
7837.280 & $2.29(52) \mathrm{E}-29$ & $\mathrm{P}(15) \mathrm{O}(16)$ \\
7844.068 & $2.87(57) \mathrm{E}-29$ & $\mathrm{P}(13) \mathrm{O}(14)$ \\
7850.698 & $3.10(93) \mathrm{E}-29$ & $\mathrm{P}(11) \mathrm{O}(12)$ \\
7857.171 & $3.49(11) \mathrm{E}-29$ & $\mathrm{P}(9) \mathrm{O}(10)$ \\
7892.018 & $1.94(8) \mathrm{E}-28$ & $\mathrm{R}(1) \mathrm{S}(0)$ \\
7895.612 & $7.64(220) \mathrm{E}-29$ & $\mathrm{R}(3) \mathrm{S}(2)$ \\
7909.781 & $1.19(18) \mathrm{E}-28$ & $\mathrm{~T}(1) \mathrm{S}(2)$ \\
7910.920 & $9.58(240) \mathrm{E}-29$ & $\mathrm{R}(9) \mathrm{S}(8)$ \\
7915.717 & $6.98(115) \mathrm{E}-29$ & $\mathrm{R}(11) \mathrm{S} 10)$ \\
\hline
\end{tabular}


Table 2. Predicted line positions and intensities for transitions with $\Delta J= \pm 2$, except $S(N) S(J)$ and $O(N) O(J)$ branches

\begin{tabular}{|c|c|c|}
\hline$v, \mathrm{~cm}^{-1}$ & $\mathrm{~S}, \mathrm{~cm} / \mathrm{molecule}$ & Assignment \\
\hline 7705.3788 & 7.37E-30 & $\mathrm{N} 21 \mathrm{O} 20$ \\
\hline 7723.9231 & $1.15 \mathrm{E}-29$ & N19O18 \\
\hline 7742.3307 & $1.66 \mathrm{E}-29$ & N17016 \\
\hline 7760.5998 & $2.20 \mathrm{E}-29$ & $\mathrm{~N} 15 \mathrm{O} 14$ \\
\hline 7778.7286 & $2.66 \mathrm{E}-29$ & $\mathrm{~N} 13 \mathrm{O} 12$ \\
\hline 7796.7154 & $2.86 \mathrm{E}-29$ & $\mathrm{~N} 11010$ \\
\hline 7808.5976 & $5.19 \mathrm{E}-30$ & $\mathrm{P} 23 \mathrm{O} 24$ \\
\hline 7814.5587 & $2.61 \mathrm{E}-29$ & N9O 8 \\
\hline 7816.0008 & $8.77 \mathrm{E}-30$ & $\mathrm{P} 21 \mathrm{O} 22$ \\
\hline 7823.2496 & $1.38 \mathrm{E}-29$ & P19020 \\
\hline 7830.3439 & $2.03 \mathrm{E}-29$ & P17018 \\
\hline 7832.2583 & $1.81 \mathrm{E}-29$ & N 706 \\
\hline 7837.2836 & $2.76 \mathrm{E}-29$ & P15O16 \\
\hline 7844.0684 & $3.46 \mathrm{E}-29$ & P13O14 \\
\hline 7849.8173 & $5.69 \mathrm{E}-30$ & $\mathrm{~N} 5 \mathrm{O} 4$ \\
\hline 7850.6977 & $3.94 \mathrm{E}-29$ & P11O12 \\
\hline 7857.1707 & $3.99 \mathrm{E}-29$ & P 9010 \\
\hline 7863.4862 & $3.45 \mathrm{E}-29$ & P 708 \\
\hline 7869.6413 & $2.27 \mathrm{E}-29$ & P 506 \\
\hline 7875.6298 & $6.80 \mathrm{E}-30$ & P 304 \\
\hline 7892.0174 & $1.96 \mathrm{E}-28$ & R1S0 \\
\hline 7895.6124 & $9.68 \mathrm{E}-29$ & R 3S 2 \\
\hline 7900.8507 & $1.03 \mathrm{E}-28$ & R 5S 4 \\
\hline 7905.9615 & $1.03 \mathrm{E}-28$ & R 7S 6 \\
\hline 7909.7810 & $1.13 \mathrm{E}-28$ & $\mathrm{~T} 1 \mathrm{~S} 2$ \\
\hline 7910.9190 & $9.41 \mathrm{E}-29$ & R 9S 8 \\
\hline
\end{tabular}




\begin{tabular}{ccc}
7915.7162 & $8.02 \mathrm{E}-29$ & $\mathrm{R} 11 \mathrm{~S} 10$ \\
7920.3493 & $6.38 \mathrm{E}-29$ & $\mathrm{R} 13 \mathrm{~S} 12$ \\
7924.8158 & $4.74 \mathrm{E}-29$ & $\mathrm{R} 15 \mathrm{~S} 14$ \\
7926.6632 & $1.24 \mathrm{E}-28$ & $\mathrm{~T} 3 \mathrm{~S} 4$ \\
7929.1137 & $3.30 \mathrm{E}-29$ & $\mathrm{R} 17 \mathrm{~S} 16$ \\
7933.2407 & $2.15 \mathrm{E}-29$ & $\mathrm{R} 19 \mathrm{~S} 18$ \\
7937.1948 & $1.32 \mathrm{E}-29$ & $\mathrm{R} 21 \mathrm{~S} 20$ \\
7940.9739 & $7.60 \mathrm{E}-30$ & $\mathrm{R} 23 \mathrm{~S} 22$ \\
7943.3445 & $1.28 \mathrm{E}-28$ & $\mathrm{~T} 5 \mathrm{~S} 6$ \\
7959.8465 & $1.24 \mathrm{E}-28$ & $\mathrm{~T} 7 \mathrm{~S} 8$ \\
7976.1715 & $1.12 \mathrm{E}-28$ & $\mathrm{~T} 9 \mathrm{~S} 10$ \\
7992.3183 & $9.44 \mathrm{E}-29$ & $\mathrm{~T} 11 \mathrm{~S} 12$ \\
8008.2844 & $7.43 \mathrm{E}-29$ & T13S14 \\
8024.0666 & $5.48 \mathrm{E}-29$ & T15S16 \\
8039.6615 & $3.80 \mathrm{E}-29$ & T17S18 \\
8055.0656 & $2.47 \mathrm{E}-29$ & T19S20 \\
8070.2751 & $1.51 \mathrm{E}-29$ & T21S22 \\
8085.2860 & $8.69 \mathrm{E}-30$ & T23S24 \\
\hline
\end{tabular}


a)

141222saaaaa.022 $\psi=82.45^{\circ} \quad \mathrm{Z}_{\mathrm{T}}=0.48 \mathrm{~km} \quad \sigma_{\mathrm{rms}}=0.5408 \% \quad \int \mathrm{dz}=4.439 \pm 0 . \mathrm{C}$

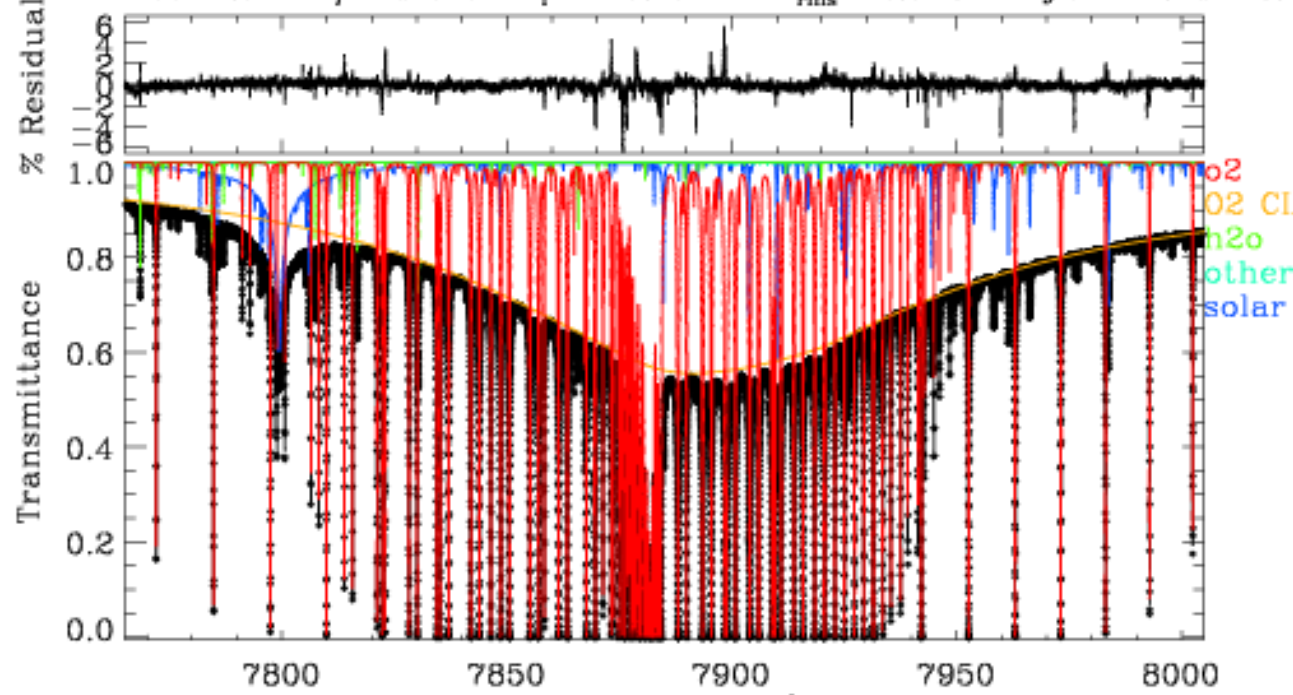

141222 saaaaa.022 $\psi=82.45^{\circ} \mathrm{Z}_{\mathrm{T}}=0.48 \mathrm{~km} \quad \sigma_{\text {rms }}=0.3162 \% \quad \int \mathrm{dz}=4.389 \pm 0 . c$
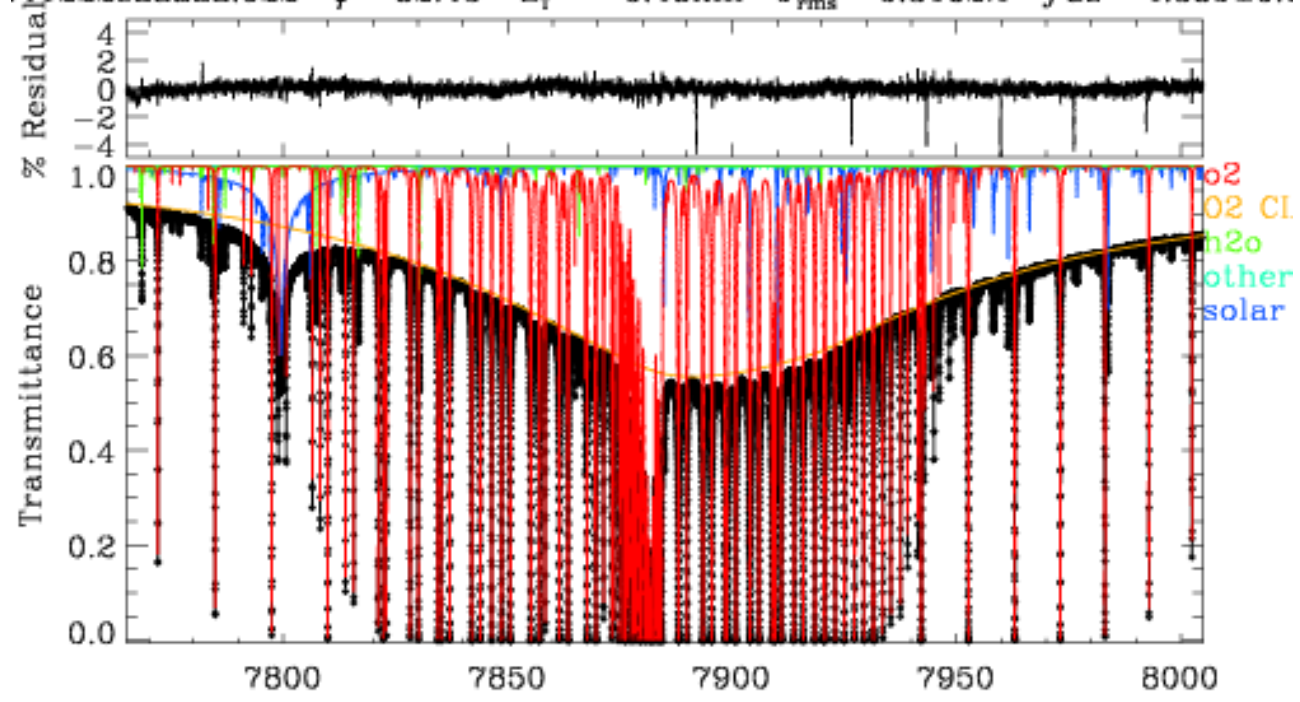

C) 141222 saaaaa.022 $\psi=82.45^{\circ} \mathrm{Z}_{\mathrm{T}}=0.48 \mathrm{~km} \quad \sigma_{\mathrm{rms}}=0.2908 \% \quad \int \mathrm{dz}=4.385 \pm 0.0$

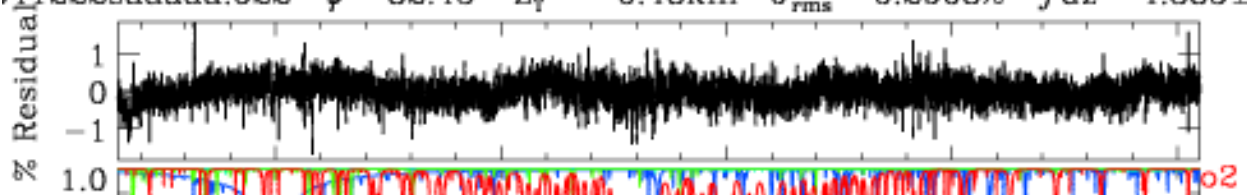

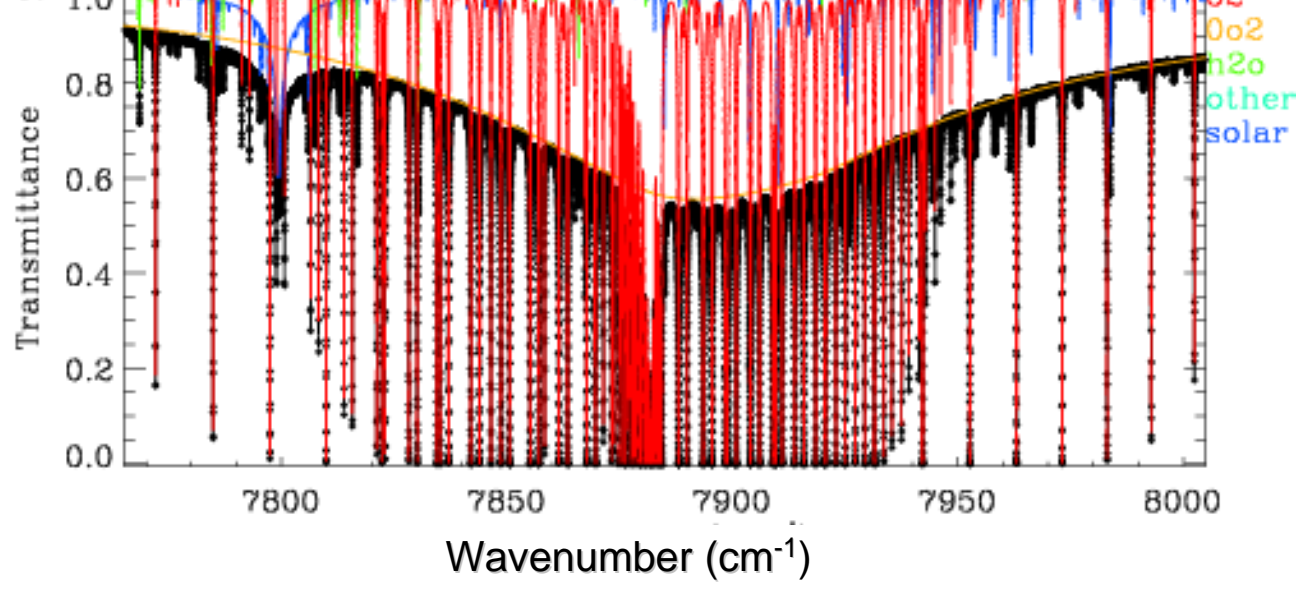




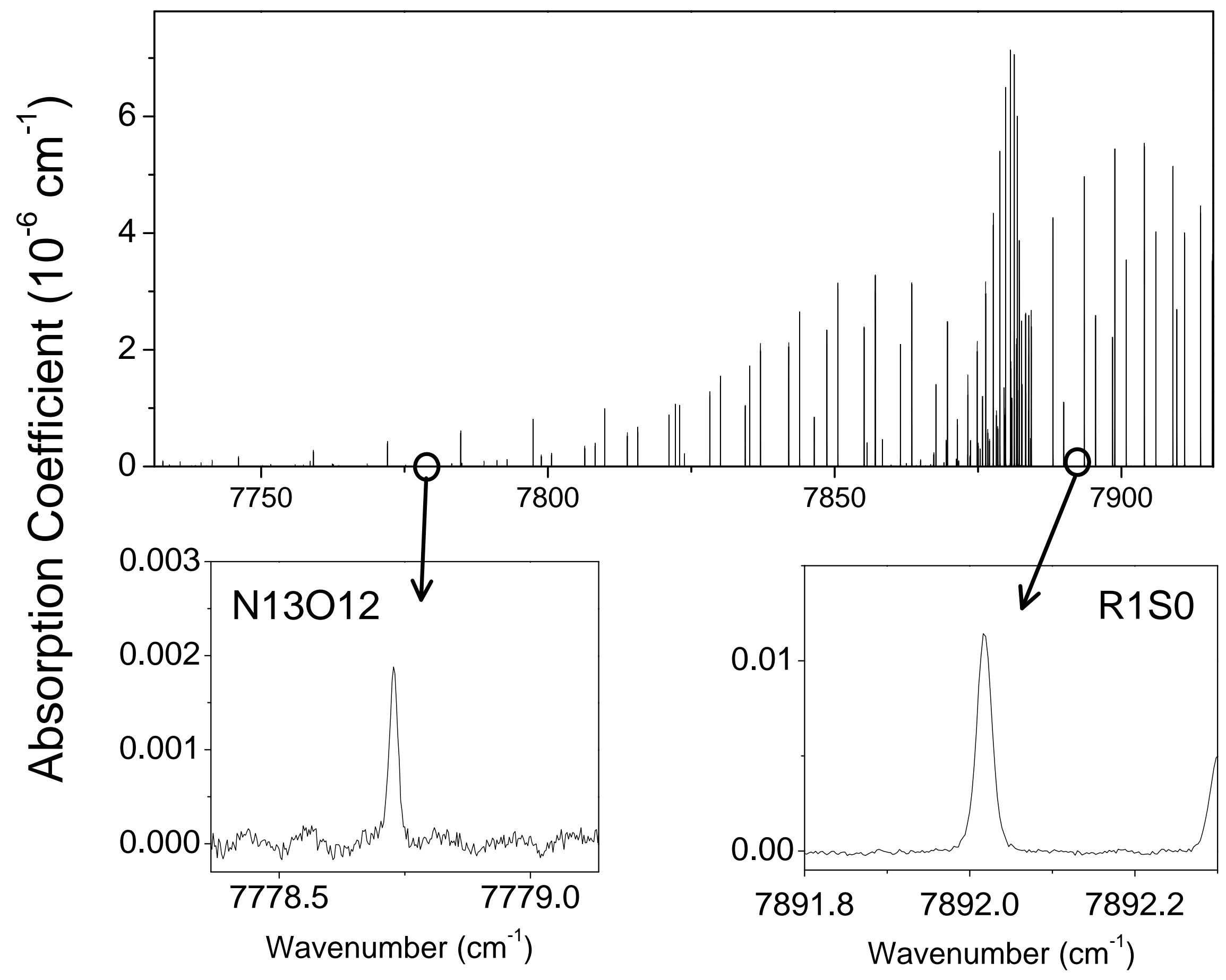




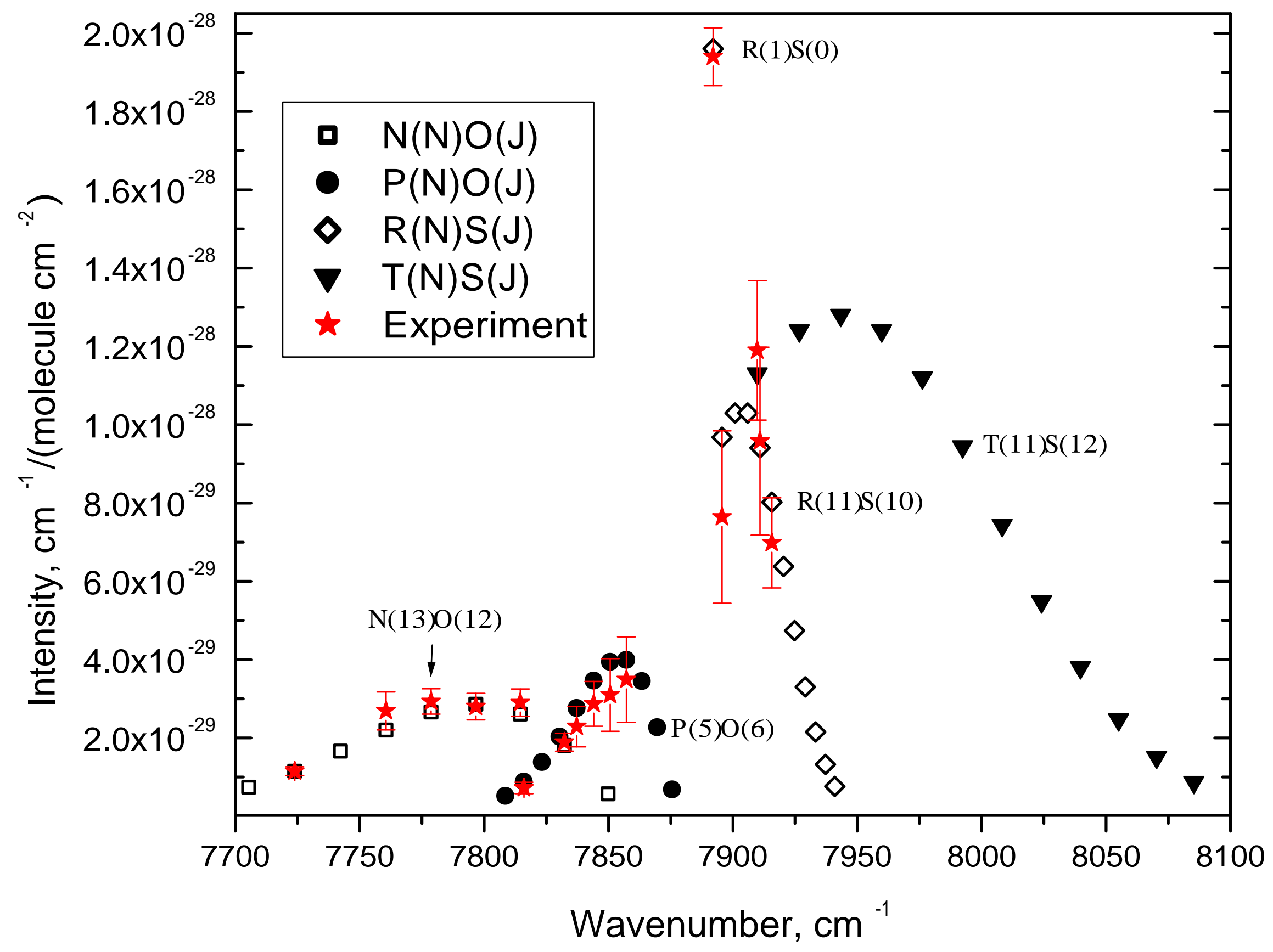




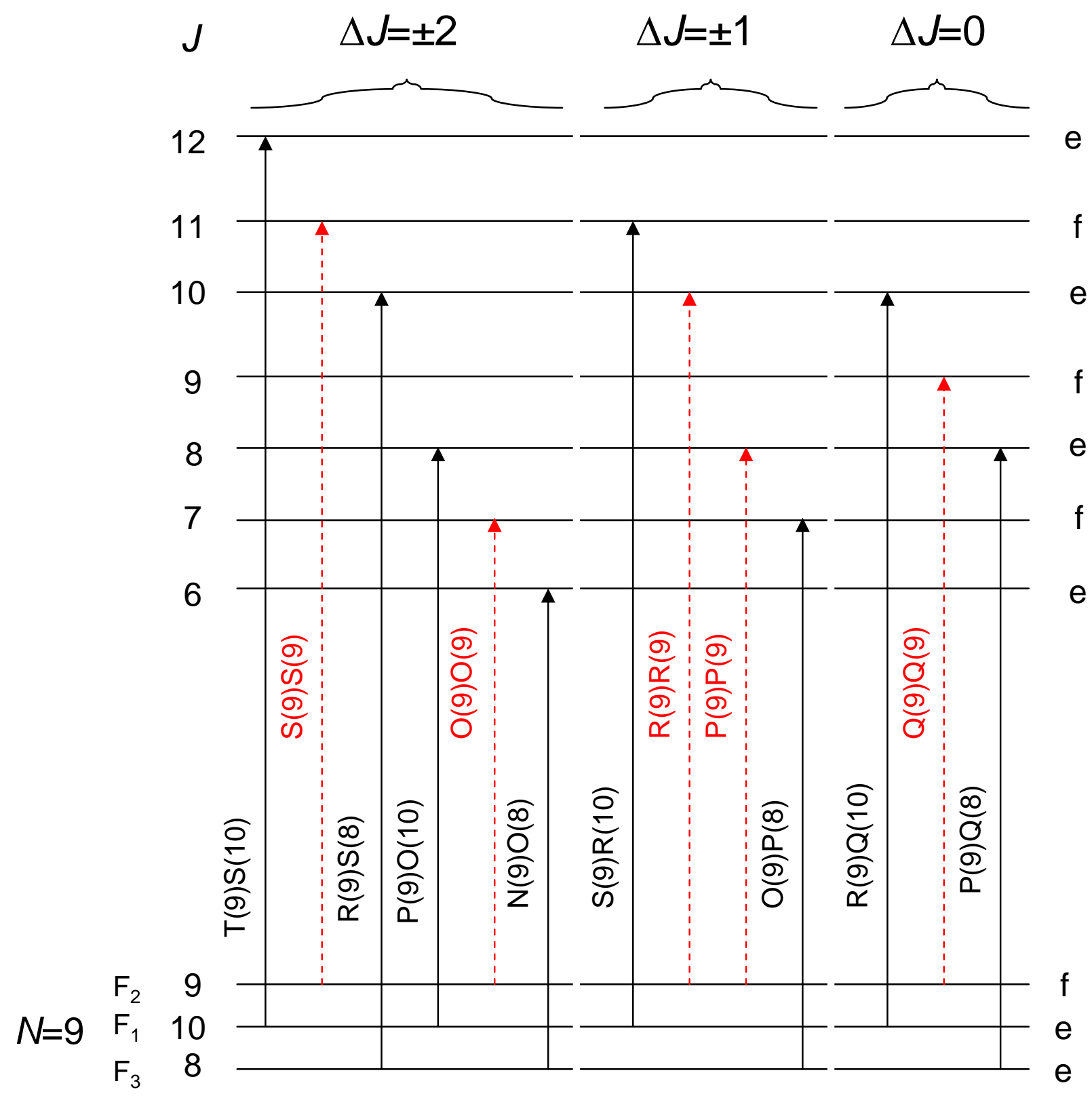



Click here to download e-component, for online publication only: supplementary.dat e-component, for online publication only
Click here to download e-component, for

(1)

